\title{
Spin susceptibilities, spin densities, and their connection to spin currents
}

\author{
Sigurdur I. Erlingsson, John Schliemann, and Daniel Loss \\ Department of Physics and Astronomy, University of Basel, Klingelbergstrasse 82, CH-4056, Switzerland
}

(Received 22 June 2004; published 14 January 2005)

\begin{abstract}
We calculate the frequency-dependent spin susceptibilities for a two-dimensional electron gas with both Rashba and Dresselhaus spin-orbit interactions. The resonances of the susceptibilities depend on the relative values of the Rashba and Dresselhaus spin-orbit constants, which could be manipulated by gate voltages. We derive exact continuity equations, with source terms, for the spin density and use those to connect the spin current to the spin density. In the free electron model the susceptibilities play a central role in the spin dynamics since both the spin density and the spin current are proportional to them.
\end{abstract}

DOI: 10.1103/PhysRevB.71.035319

PACS number(s): 73.63. $-\mathrm{b}, 85.75 .-\mathrm{d}$

\section{INTRODUCTION}

The ability to manipulate spin states in semiconducting and metallic nanostructures is now the focus of much experimental and theoretical attention. Some spintronics application are already in use $\mathrm{e}^{1,2}$ and more have been proposed, ranging from spin field-effect transistors ${ }^{3-5}$ to spin qubits. ${ }^{6,7}$ In many cases the spin-orbit interaction can be used to manipulate the electron spin via electronic means. ${ }^{8}$ This sort of electronic manipulation is important since technologically, electric field control of spins is preferred over magnetic field control. Also, electronic control of spins has revealed many interesting physics in experiments involving semiconductor heterostructures. ${ }^{9,10}$

Some time ago it was proposed that electric fields could lead to magnetization in antiferromagnetic materials fulfilling certain symmetries. ${ }^{11}$ This is the so-called magnetoelectric effect. The magnetoelectric effect was considered for conductors with special symmetry properties ${ }^{12}$ and for pyroelectric superconductors. ${ }^{13}$ Electric-field-induced spin orientation in semiconductors due to linear in momentum spinorbit interaction was also discussed in Refs. 14-16. There has been renewed interest in this subject since this induced spin polarization might serve as spin injectors in certain semiconductor heterostructures. ${ }^{17,18}$ Only recently there have been experiments which seem to demonstrate such current induced polarization of spins via spin-orbit interaction. ${ }^{19,20}$

A somewhat related effect is the so-called spin-Hall effect. In the normal Hall effect the electrons are deflected by the Lorentz force, but in the spin-Hall effect spins are scattered by impurities preferentially to the left (right) if their spins point "up" ("down") due to spin-orbit interaction. ${ }^{21,22}$ This will result in a spin current, but no net charge current, flowing perpendicular to the applied charge current. Such an extrinsic transverse spin current was already investigated by Dyakononov and Perel some time ago in Ref. 23 and more recently by Hirsch. ${ }^{24}$ Presently there is much interest in spinorbit mediated spin-Hall effect in semiconducting heterostructures. Here the effect can appear in hole- ${ }^{25-31}$ or electron-doped ${ }^{32-42}$ semiconductors due to band structure properties and impurities are not necessary. Thus, the term intrinsic spin Hall effect is used to distinguish this from the previously discussed mechanism since it occurs already in the absence of impurities. ${ }^{26,32}$ Due to differences in the band structure of the holes and electron there is an important distinction between the two since in the electron system the spin Hall conductance (the ratio of the transverse spin current and the applied electric field) reaches a universal value of $e / 8 \pi$ in clean systems. ${ }^{32}$ However, impurities are believed to modify this universal value ${ }^{33,40}$ and in addition, the exact influence of vertex corrections on the spin-Hall conductivity is currently under investigation. ${ }^{38,42,43}$

In this paper we consider the spin susceptibilities of a 2DEG with Rashba and Dresselhaus spin-orbit coupling. Due to the strong 2DEG confinement the Dresselhaus coupling reduces to terms linear in momentum. For such linear momentum spin-orbit coupling the spin susceptibility can be used to characterize other transport properties. We calculate the susceptibilities using a free electron model and relate them to the electric-field-induced spin density. Also, we derive continuity equations (with source terms) for the spin density and spin current, similar to the equations already derived for only Rashba coupling. ${ }^{36}$ These equations are exact operator identities and via them we can relate the spin current to the spin density. Via these relations the spin current (which is nontrivial to measure) can be connected to the spin density, or magnetization, which is easier to detect. The susceptibilites play a central role in the free electron model, since the Fourier transform of the spin density and the spin current are proportional to the susceptibilities.

\section{THE MODEL}

We consider a two-dimensional electron gas (2DEG) with Rashba and Dresselhaus spin-orbit coupling. In the absence of external fields the Hamiltonian may be written as

$$
H=\frac{p_{x}^{2}+p_{y}^{2}}{2 m}+\frac{\alpha}{\hbar}\left(p_{y} \sigma_{x}-p_{x} \sigma_{y}\right)+\frac{\beta}{\hbar}\left(p_{y} \sigma_{y}-p_{x} \sigma_{x}\right),
$$

where $\alpha$ and $\beta$ are the Rashba and Dresselhaus (linear) coefficient in a 2DEG. It is easy to see that $\left[p_{x}, H\right]=\left[p_{y}, H\right]$ $=0$, and thus we seek eigenstates of the form

$$
\psi_{\boldsymbol{k}, s}(\boldsymbol{r}) \equiv\langle\boldsymbol{r} \mid \boldsymbol{k} s\rangle=\frac{e^{i \boldsymbol{k} \cdot \boldsymbol{r}}}{\sqrt{A}} \boldsymbol{u}_{s}(\boldsymbol{k})
$$

where $\boldsymbol{u}_{s}(\boldsymbol{k})$ is spinor to be determined and $A$ is the system area. Since the momenta are conserved it is possible to introduce an effective magnetic field 


$$
\boldsymbol{\Gamma}=\left(\begin{array}{c}
-\beta k_{x}+\alpha k_{y} \\
\beta k_{y}-\alpha k_{x} \\
0
\end{array}\right)
$$

The eigenspectrum can be written in terms of this effective magnetic field, the eigenenergies of the Hamiltonian being $(s= \pm 1)$

$$
E_{s}(\boldsymbol{k})=\frac{\hbar^{2} k^{2}}{2 m}+s\left|\Gamma^{+}\right|
$$

and the corresponding spinor

$$
\boldsymbol{u}_{s}(\boldsymbol{k})=\frac{1}{\sqrt{2}}\left(\begin{array}{c}
1 \\
s \frac{\Gamma^{+}}{\left|\Gamma^{+}\right|}
\end{array}\right)
$$

where $\Gamma^{+}=\Gamma_{x}+i \Gamma_{y}$. Note that Eqs. (4) and (5) are also valid for an in-plane magnetic field $\boldsymbol{B}$ using the substitution $\boldsymbol{\Gamma}$ $\rightarrow \boldsymbol{\Gamma}+\frac{1}{2} g \mu_{B} \boldsymbol{B}$, where $g$ and $\mu_{B}$ are the effective $g$-factor and Bohr magneton, respectively.

Writing the quasimomentum in polar coordinates $\boldsymbol{k}$ $=k(\cos \theta, \sin \theta)$, the eigenspectrum of the Hamiltonian in Eq.

(1) becomes

$$
E_{s}(\boldsymbol{k})=\frac{\hbar^{2} k^{2}}{2 m}+s k \sqrt{\left(\alpha^{2}+\beta^{2}\right) g(\theta)},
$$

where $g(\theta)=1-\sin (2 \phi) \sin (2 \theta)$ determines the anisotropy of the Fermi surfaces and the corresponding eigenspinors are

$$
\boldsymbol{u}_{s}(\boldsymbol{k})=\frac{1}{\sqrt{2}}\left(\begin{array}{c}
1 \\
-s \frac{\cos (\phi) e^{-i \theta}+i \sin (\phi) e^{i \theta}}{g(\theta)}
\end{array}\right) .
$$

Here we have introduced the following parametrization of the spin-orbit coupling strength:

$$
\sin (\phi)=\frac{\alpha}{\sqrt{\alpha^{2}+\beta^{2}}}, \quad \phi \in[-\pi / 2, \pi / 2] .
$$

The eigenfunctions in Eq. (7) have the interesting property that they depend on the spin-orbit coupling parameters $\alpha$ and $\beta$ only via the angle $\phi$. This allows one to take the limit $\alpha, \beta \rightarrow 0$ such that the angle $\phi$ remains fixed and the resulting eigenvectors in Eq. (9) are also (degenerate) eigenvectors of the free electron Hamiltonian. ${ }^{44}$ As was pointed out in Ref. 37, the Kramers conjugate state of $\psi_{\boldsymbol{k}, s}(\boldsymbol{r})$ is $\psi_{-\boldsymbol{k}, s}(\boldsymbol{r})$, i.e., they belong to the same branch.

\section{SPIN SUSCEPTIBILITIES AND CURRENT-INDUCED MAGNETIZATION}

For a weak driving field the response of the system is obtained by the Kubo formalism. Due to the spin-orbit coupling, a pure electric field driving results in a nonzero magnetic response. Since the spin-orbit term in Eq. (1) is linear in momenta, both the response functions due to magnetic and electric perturbation can be expressed with spin susceptibilities. The $\eta=x, y, z$ component of the spin density operator is defined as

$$
\rho_{\eta}(\boldsymbol{r})=\sum_{n} \sigma_{n, \eta}(\boldsymbol{r})=\sum_{n} \sigma_{n, \eta} \delta\left(\boldsymbol{r}-\boldsymbol{r}_{n}\right)
$$

where $\boldsymbol{r}_{n}$ and $\sigma_{n, \eta}$ are the position operator and Pauli matrix, respectively, of the $n$th electron. For a translationally invariant system, the wave vector and frequency $(\boldsymbol{q}, \omega)$ dependent susceptibilities are

$$
\begin{gathered}
\chi_{\eta \eta^{\prime}}(\boldsymbol{q}, \omega)=\int_{0}^{\infty} d t e^{i \omega t} \frac{i}{\hbar A}\left\langle\left[\rho_{\eta}(\boldsymbol{q}, t), \rho_{\eta^{\prime}}(-\boldsymbol{q})\right]\right\rangle, \\
=\int_{0}^{\infty} d t e^{i \omega t} \frac{1}{A} \sum_{\boldsymbol{k}, s} \frac{i f_{\boldsymbol{k} s}}{\hbar}\left\langle\left[\sigma_{\eta}(\boldsymbol{q}, t), \sigma_{\eta^{\prime}}(-\boldsymbol{q})\right]\right\rangle_{\boldsymbol{k} s},
\end{gathered}
$$

where $f_{\boldsymbol{k} s}=f\left[E_{s}(\boldsymbol{k})\right]$ with $f$ being the Fermi distribution function and we used the notation $\langle\cdots\rangle_{\boldsymbol{k} s}=\langle\boldsymbol{k} s|\cdots| \boldsymbol{k} s\rangle$. The frequency should be viewed as $i \omega \rightarrow i\left(\omega+i \hbar^{-1} \epsilon\right)$ to regularize the integral. The operators in Eq. (11) refer to single particle operators. This susceptibility is a spin density response function and to get the magnetization response function, each spin density operator in Eq. (11) should be multiplied with the electron effective magnetic moment $g \mu_{B} / 2$. Using Eq. (11) and the eigenspectrum represented by Eqs. (6) and (7) the susceptibilities for a spatially homogeneous perturbation $(\boldsymbol{q}=0)$ become

$$
\begin{aligned}
& \chi_{x x}(\omega)= \frac{1}{(2 \pi)^{2} \hbar} \int_{0}^{2 \pi} d \theta \frac{(\alpha \sin \theta-\beta \cos \theta)^{2}}{\left(\alpha^{2}+\beta^{2}\right)^{1 / 2} \sqrt{g(\theta)}} \int_{k_{+}(\theta)}^{k_{-}(\theta)} d k \\
& \times \frac{k^{2}}{4\left(\alpha^{2}+\beta^{2}\right) g(\theta) k^{2}-(\hbar \omega+i \epsilon)^{2}}, \\
& \chi_{x y}(\omega)= \frac{1}{(2 \pi)^{2} \hbar} \int_{0}^{2 \pi} d \theta \frac{(\alpha \sin \theta-\beta \cos \theta)(\beta \sin \theta-\alpha \cos \theta)}{\left(\alpha^{2}+\beta^{2}\right)^{1 / 2} \sqrt{g(\theta)}} \\
& \times \int_{k_{+}(\theta)}^{k_{-}(\theta)} d k \frac{k^{2}}{4\left(\alpha^{2}+\beta^{2}\right) g(\theta) k^{2}-(\hbar \omega+i \epsilon)^{2}} .
\end{aligned}
$$

Note that all $\boldsymbol{q}=0$ dependence has been dropped for clarity. Here we assumed zero temperature and the Fermi distribution function was replaced by a step function. The resulting Fermi contours $k_{ \pm}(\theta)$ are the solutions of

$$
k_{F}^{2}=k_{ \pm}(\theta)^{2} \pm k_{ \pm}(\theta) k_{\mathrm{SO}} \sqrt{g(\theta)},
$$

where $k_{E}^{2}=2 m \epsilon_{F} / \hbar^{2}$ is the squared Fermi momentum and $k_{\mathrm{SO}}=m \sqrt{\alpha^{2}+\beta^{2}} / \hbar^{2}$. The $k$ integral results in a linear term and a term involving an inverse tangent in $k_{ \pm}(\theta)$ and the subsequent angular integrals cannot be solved analytically. The inverse tangent can be expanded in powers of $k_{\mathrm{SO}} / k_{F} \ll 1$, resulting in the following lowest order result:

$$
\chi_{x x}(\omega)=\chi_{y y}(\omega)=\frac{m}{2 \pi \hbar^{2}}\left(1+\frac{(\hbar \omega+i \epsilon)^{2}}{\prod_{s} \sqrt{\varepsilon_{s}^{2}-(\hbar \omega+i \epsilon)^{2}}}\right),
$$

where the resonance energies are $\epsilon_{ \pm}^{2}=8 \epsilon_{\mathrm{SO}} \epsilon_{F}[1 \pm \sin (2 \phi)]$, with $\epsilon_{\mathrm{SO}}=m\left(\alpha^{2}+\beta^{2}\right) / \hbar^{2}$. Using the same procedure we can calculate the off-diagonal susceptibilities in a similar manner 


$$
\chi_{x y}(\omega)=\chi_{y x}(\omega)=\frac{\delta \chi(\omega)-\chi_{x x}(\omega)}{\sin (2 \phi)}-\sin (2 \phi) \delta \chi(\omega),
$$

where we have defined

$$
\delta \chi(\omega)=\frac{m}{2 \pi \hbar^{2}} \frac{8 \varepsilon_{\mathrm{SO}} \varepsilon_{F}}{\prod_{s} \sqrt{\varepsilon_{s}^{2}-(\hbar \omega+i \epsilon)^{2}}} .
$$

The magnetization is related to the spin density in Eq. (9) through $\boldsymbol{m}(\boldsymbol{r})=\frac{1}{2} g \mu_{B} \boldsymbol{\rho}(\boldsymbol{r})$ which leads to the standard linear response relation

$$
m_{\eta}(\boldsymbol{q}, \omega)=\left(\frac{1}{2} g \mu_{B}\right)^{2} \chi_{\eta \eta^{\prime}}(\boldsymbol{q}, \omega) B_{\eta^{\prime}}(\boldsymbol{q}, \omega) .
$$

To obtain the Pauli paramagnetic susceptibility one should take the following order of limits: ${ }^{45}$

$$
\lim _{\boldsymbol{q} \rightarrow 0} \lim _{\omega \rightarrow 0} \chi_{\eta \eta^{\prime}}(\boldsymbol{q}, \omega)=\frac{m}{\pi \hbar^{2}} \delta_{\eta, \eta^{\prime}} .
$$

The diamagnetic contribution can be disregarded since we assume an in-plane magnetic field. The susceptibilities in Eqs. (12) and (13) are calculated for the reverse order of limits done in Eq. (19). These $\omega \neq 0$ susceptibilities are the spin-orbit contribution coming from the region in $\boldsymbol{k}$ space where only one $(s=-1)$ of the two branches is occupied. ${ }^{55}$ Also, the spin current (see discussion below) which results from the spin-orbit interaction is nonzero due to contributions from the $\boldsymbol{k}$ space area between the two Fermi contours. $^{32,33,41}$ Thus we only focus on this contribution when we relate the susceptibilities to the spin-orbit mediated spin densities and the spin currents.

The value of the resonance frequency is determined by $\sqrt{8 \varepsilon_{\mathrm{SO}} \varepsilon_{F}} \approx 0.16 \mathrm{meV}=40 \mathrm{GHz}$ for typical GaAs parameters: ${ }^{46} \quad \alpha=0.5 \times 10^{-9} \mathrm{meV} \mathrm{m}$ and electron density $n_{e}=4$ $\times 10^{15} \mathrm{~m}^{-2}$. For lower frequencies the susceptibilities remain nominally constant. In the limit $\alpha \ll \beta$ the lowest order contribution to Eqs. (15) and (16) become

$$
\begin{gathered}
\chi_{x x}(\omega)=\frac{m}{2 \pi \hbar^{2}} \frac{1}{1-\frac{(\hbar \omega+i \epsilon)^{2}}{8 \varepsilon_{\mathrm{SO}} \varepsilon_{F}}}, \\
\chi_{x y}(\omega)=-\frac{m}{2 \pi \hbar^{2}} \frac{\alpha}{\beta} \frac{1-\frac{(\hbar \omega+i \epsilon)^{2}}{4 \varepsilon_{\mathrm{SO}} \varepsilon_{F}}}{\left(1-\frac{(\hbar \omega+i \epsilon)^{2}}{8 \varepsilon_{\mathrm{SO}} \varepsilon_{F}}\right)^{2}} .
\end{gathered}
$$

Here we have not included impurities and thus the regularization parameter $\epsilon$ can strictly only be attributed to an adiabatic turning on of the external electric or magnetic field.

In the absence of electric and magnetic fields, the spinorbit interaction does not give rise to a net magnetization. Even though the spin-orbit interaction has the form of a momentum-dependent magnetic field, the total contribution averages to zero. ${ }^{47,48}$ However, although the spin-orbit induced splitting does not give rise to an equilibrium magne- tization, there is an asymmetry in the local magnetic field in momentum space, i.e., the local magnetic field is odd under wave vector reversal, and any translation of the Fermi sphere away from the $\Gamma$ point will induce a magnetization. ${ }^{15}$ Applying a homogeneous electric field $\boldsymbol{E}(\boldsymbol{r}, t)=\boldsymbol{E}^{0} e^{-i \omega t}$ to the system will give rise to the following time-dependent perturbation:

$$
V(t)=-\frac{e}{i \omega} \boldsymbol{E}^{0} e^{-i \omega t} \cdot \boldsymbol{j}(\boldsymbol{q}=0),
$$

where $\boldsymbol{j}(\boldsymbol{q})$ is the Fourier transform of the current density operator

$$
\boldsymbol{j}(\boldsymbol{r})=\sum_{n} \boldsymbol{j}_{n}(\boldsymbol{r})=\sum_{n} \frac{1}{2}\left\{\delta\left(\boldsymbol{r}-\boldsymbol{r}_{n}\right), \boldsymbol{v}_{n}\right\},
$$

and the velocity operator $\boldsymbol{v}_{n}$ for the Hamiltonian in Eq. (1) is given by

$$
\boldsymbol{v}_{n}=\left(\begin{array}{l}
\frac{1}{m} p_{n, x}-\frac{\alpha}{\hbar} \sigma_{n, y}-\frac{\beta}{\hbar} \sigma_{n, x} \\
\frac{1}{m} p_{n, y}+\frac{\alpha}{\hbar} \sigma_{n, x}+\frac{\beta}{\hbar} \sigma_{n, y}
\end{array}\right) .
$$

From linear response theory the Fourier transform of the electric field induced spin density may be written as

$$
\begin{aligned}
\left\langle\rho_{x}(\omega)\right\rangle= & \frac{e E_{x}(\omega)}{i \omega}\left(\frac{\alpha}{\hbar} \chi_{x y}(\omega)+\frac{\beta}{\hbar} \chi_{x x}(\omega)\right) \\
& -\frac{e E_{y}(\omega)}{i \omega}\left(\frac{\alpha}{\hbar} \chi_{x x}(\omega)+\frac{\beta}{\hbar} \chi_{x y}(\omega)\right), \\
\left\langle\rho_{y}(\omega)\right\rangle= & \frac{e E_{x}(\omega)}{i \omega}\left(\frac{\alpha}{\hbar} \chi_{x x}(\omega)+\frac{\beta}{\hbar} \chi_{x y}(\omega)\right) \\
& -\frac{e E_{y}(\omega)}{i \omega}\left(\frac{\alpha}{\hbar} \chi_{x y}(\omega)+\frac{\beta}{\hbar} \chi_{x x}(\omega)\right) .
\end{aligned}
$$

This result is reminiscent of the pure magnetic field induced spin density, except here $e E_{y}(\omega) / i \omega$ plays the role of magnetic field, via the spin-orbit coupling. As was pointed out in Refs. 15 and 41 the dc limit corresponds to replacing the frequency with momentum scattering $i \omega \rightarrow-1 / \tau$.

Multiplying Eqs. (25) and (26) by the sample area will give the total number of induced magnetic moments, measured in units of $g \mu_{B} / 2$. Applying an electric field $E$ $\approx 100 \mathrm{~V} / \mathrm{cm}$ to a GaAs 2DEG's with high mobility $(\hbar / \tau$ $\left.\approx 10^{-2} \mathrm{meV}\right)$ and a sample area of $A=(500 \mu \mathrm{m})^{2}$, the number of magnetic moments (Bohr magnetons) would be around $2.5 \times 10^{7}$. For a 2 DEG thickness of a few nm these magnetic moments produce a magnetic field of the order $10^{-6} \mathrm{~T}$. Probing $2 \mathrm{DEG}$ properties using ESR techniques has been succesfully used to determine the spin-orbit splitting ${ }^{49}$ and other 2DEG spin properties, ${ }^{50,51}$ Using similar ESR techniques, the spin-orbit coefficients $\alpha, \beta$ could in principle be determined by measuring different spin density component for different direction of driving current, as a function of $\alpha$ 
which could be tuned by gate voltages. ${ }^{46,52,53}$ Such an induced spin density could be detected by Faraday rotation measurements. ${ }^{19}$

\section{CONNECTION TO SPIN CURRENT}

The usual way of deriving the operator version of the particle continuity equation is to start from the definition of the density operator

$$
\rho(\boldsymbol{r}, t)=\sum_{n} \delta\left[\boldsymbol{r}-\boldsymbol{r}_{n}(t)\right],
$$

and from there one can derive the standard continuity equation,

$$
\frac{\partial}{\partial t} \rho(\boldsymbol{r}, t)+\nabla \cdot \boldsymbol{j}(\boldsymbol{r}, t)=0
$$

from the Heisenberg equation of motion for $\rho(\boldsymbol{r}, t)$. The form of the current density in Eq. (28) is uniquely determined by the Heisenberg equation of motion. For the Hamiltonian in Eq. (1) the current density is given by Eq. (23), using the velocity operator in Eq. (24). In the case of the spin density operator

$$
\rho_{\eta}(\boldsymbol{r}, t)=\sum_{n} \sigma_{n, \eta}(t) \delta\left[\boldsymbol{r}-\boldsymbol{r}_{n}(t)\right]
$$

the same procedure of evaluating the Heisenberg equation motion will not result in a unique definition of the associated spin current density. The reason is that the precession due to the momentum dependent spin-orbit magnetic field introduces additional terms into the equation of motion. In order to proceed one has to postulate a form for the spin current density. The most widely used definition, and physically reasonable, is the following:

$$
\boldsymbol{j}^{\eta}(\boldsymbol{r})=\sum_{n} \frac{1}{2}\left\{\sigma_{n, \eta, j_{n}}(\boldsymbol{r})\right\} .
$$

This form of the spin current is Hermitian and reduces to the standard spin current form when the velocity operator is spin independent. ${ }^{54}$ Having determined the form of the spin current the resulting continuity equations become

$$
\begin{aligned}
\frac{\partial}{\partial t} \rho_{x}(\boldsymbol{r}, t)+\nabla \cdot \boldsymbol{j}^{x}(\boldsymbol{r}, t)= & -\frac{2 m \alpha}{\hbar^{2}} j_{x}^{z}(\boldsymbol{r}, t)+\frac{2 m \beta}{\hbar^{2}} j_{y}^{z}(\boldsymbol{r}, t), \\
\frac{\partial}{\partial t} \rho_{y}(\boldsymbol{r}, t)+\nabla \cdot \boldsymbol{j}^{y}(\boldsymbol{r}, t)= & -\frac{2 m \alpha}{\hbar^{2}} j_{y}^{z}(\boldsymbol{r}, t)+\frac{2 m \beta}{\hbar^{2}} j_{x}^{z}(\boldsymbol{r}, t), \\
\frac{\partial}{\partial t} \rho_{z}(\boldsymbol{r}, t)+\nabla \cdot j^{z}(\boldsymbol{r}, t)= & +\frac{2 m \alpha}{\hbar^{2}}\left[j_{y}^{x}(\boldsymbol{r}, t)+j_{x}^{y}(\boldsymbol{r}, t)\right] \\
& -\frac{2 m \beta}{\hbar^{2}}\left[j_{x}^{x}(\boldsymbol{r}, t)+j_{y}^{y}(\boldsymbol{r}, t)\right] .
\end{aligned}
$$

Similar equations have already been derived for pure Rashba coupling. ${ }^{36}$ The above Eqs. (31)-(33) are exact relation for a systems with spin-orbit coupling linear in momentum and including impurities would not change their form. ${ }^{56,57}$

Taking the thermal average of Eqs. (31)-(33) gives partial differential equation connecting the spin densities and spin currents. Based on these equations one can make a few observation on the nature of the spin current, without explicitly solving them. First of all, for a homogeneous system in the stationary limit the right-hand side of Eq. (33) must vanish for all values of $\alpha, \beta$. This is trivially satisfied for all $\left\langle j^{\eta}\right\rangle$ $=0$, but more interestingly also when $\left\langle j_{y}^{x}\right\rangle=-\left\langle j_{x}^{y}\right\rangle$ and $\left\langle j_{x}^{x}\right\rangle=$ $-\left\langle j_{y}^{y}\right\rangle$. It is easy to show that the latter case is true in equilibrium

$$
\begin{aligned}
& \left\langle j_{x}^{x}\right\rangle=-\left\langle j_{y}^{y}\right\rangle=\frac{1}{3 \pi} \frac{\beta}{\hbar}\left(\frac{m}{\hbar^{2}}\right)^{2}\left(\alpha^{2}-\beta^{2}\right), \\
& \left\langle j_{x}^{y}\right\rangle=-\left\langle j_{y}^{x}\right\rangle=\frac{1}{3 \pi} \frac{\alpha}{\hbar}\left(\frac{m}{\hbar^{2}}\right)^{2}\left(\alpha^{2}-\beta^{2}\right),
\end{aligned}
$$

which covers the results of Ref. 37 as special cases. In particular, the current expectation values vanish at $\alpha= \pm \beta$ due to the additional concerved quantity arising at these points. ${ }^{4}$ Furthermore, Eq. (33) shows these equilibrium currents do not act as source terms for the spin density, since the rhs always vanishes.

Let us now consider a homogeneous system such that the divergence terms vanish. By using Eqs. (31) and (32) one can derive the following identity for the Fourier transform of the $x$ and $y$ component of the average $z$-polarized spin current:

$$
\begin{aligned}
\left\langle j_{x}^{z}(\omega)\right\rangle & =\frac{\hbar^{2} i \omega\left[\alpha\left\langle\rho_{x}(\omega)\right\rangle+\beta\left\langle\rho_{y}(\omega)\right\rangle\right]}{2 m\left(\beta^{2}-\alpha^{2}\right)}, \\
\left\langle z_{y}^{z}(\omega)\right\rangle & =\frac{\hbar^{2} i \omega\left[\beta\left\langle\rho_{x}(\omega)\right\rangle+\alpha\left\langle\rho_{y}(\omega)\right\rangle\right]}{2 m\left(\beta^{2}-\alpha^{2}\right)} .
\end{aligned}
$$

These relations establish a connection between the spin current components $\left\langle j_{x, y}^{z}\right\rangle$ and the $x, y$ components of the spin density, in the frequency domain. This is quite useful since the spin current, which is hard to detect, is related to a quantity which is easier to measure. Also, Eqs. (36) and (37) is a good starting point for connecting spin current and spin density response functions using standard Kubo formalism.

Let us now assume a homogeneous electric field applied in the $y$ direction. The spin conductivities are defined as the ratio of the spin current and applied electric field

$$
\sigma_{\eta y}^{z}(\omega)=\frac{\frac{\hbar}{2}\left\langle j_{\eta}^{z}(\omega)\right\rangle}{E_{y}(\omega)},
$$

where the factor $\hbar / 2$ in the definition of the spin conductivity is due to our definition of the spin current in terms Pauli matrices and not the spin operators, i.e., $S=\hbar \boldsymbol{\sigma} / 2$. Using Eqs. (25) and (26) to relate the spin density to the susceptibilities we obtain the following result for the ac spin conductivities: 


$$
\begin{gathered}
\sigma_{x y}^{z}(\omega)=\frac{e \hbar^{2}}{4 m} \frac{\alpha^{2}-\beta^{2}}{\beta^{2}+\alpha^{2}} \delta \chi(w), \\
\sigma_{y y}^{z}(\omega)=-\frac{\alpha^{2}-\beta^{2}}{2 \beta \alpha} \frac{e \hbar^{2}}{4 m}\left[\delta \chi(w)-\chi_{x x}(w)\right] .
\end{gathered}
$$

These equations for the spin conductivities, along with Eqs. (15) and (17), in the $\omega \rightarrow 0$ limit reproduce the result in Ref. 34 up to a sign convention for $\beta$. For either pure Rashba or Dresselhaus we have the following result:

$$
\begin{gathered}
\sigma_{x y}^{z}(\omega)= \pm \frac{e}{8 \pi}\left(\frac{1}{1-\frac{(\hbar \omega+i \epsilon)^{2}}{8 \epsilon_{\mathrm{SO}} \varepsilon_{F}}}\right), \\
\sigma_{y y}^{z}(\omega)=0
\end{gathered}
$$

the upper (lower) sign refers to a pure Rashba (Dresselhaus). Taking the limit $\epsilon \rightarrow 0$ recovers the universal limit of spin Hall conductance $\sigma_{x y}^{z}(\omega \rightarrow 0)=e / 8 \pi .^{32}$

\section{CONCLUSION}

We calculated the frequency-dependent spin susceptibilities for a 2DEG with both Rashba and Dresselhaus spin-orbit coupling. The suspectibilities have resonance peaks whose position depends on the relative magnitude of the Rashba and Dresselhaus coefficients. The position of the resonance could be tuned via the gate dependence of $\alpha$. We derive a connection between spin density and the spin current starting from the Heisenberg equation of motion for the spin density. Unlike the particle density, the resulting continuity equations have spin current source terms due to the spin-orbit interaction. Using these relation we connect the spin current to the spin density. In the free electron model the spin current and the spin-Hall coefficient are proportional to the susceptibility. Indeed, using the continuity equations and the susceptibilities calculated here we recover the universal (ballistic) value of the spin-Hall conductance. Such relations [Eqs. (36) and (37)] might help elucidate the nature of spin currents in a similar way to Ref. 41 which discussed the relation between the spin current and the dielectric function. By calculating the spin density with the correct impurity contribution would automatically give the spin current. We are confident that these and similar considerations will contribute to a deeper understanding of the role of impurities in the spin-Hall effect.

\section{ACKNOWLEDGMENTS}

The authors acknowledge financial support from the NCCR Nanoscience, the Swiss NSF, DARPA, ARO, ONR, and the Spintronics RTN. We would like to thank J. Carlos Egues, Daniel Saraga, and Oleg Chalaev for enlightening discussions.
${ }^{1}$ S. A. Wolf, D. D. Awschalom, R. A. Buhrman, J. M. Daughton, S. von Molnár, M. L. Roukes, A. Y. Chtchelkanova, and D. M. Treger, Science 294, 1488 (2001).

${ }^{2}$ G. A. Prinz, Science 282, 1660 (1998).

${ }^{3}$ S. Datta and B. Das, Appl. Phys. Lett. 56, 665 (1990).

${ }^{4}$ J. Schliemann, J. C. Egues, and D. Loss, Phys. Rev. Lett. 90, 146801 (2003).

${ }^{5}$ J. C. Egues, G. Burkard, and D. Loss, Appl. Phys. Lett. 82, 2658 (2003).

${ }^{6}$ D. Loss and D. P. DiVincenzo, Phys. Rev. A 57, 120 (1998).

${ }^{7}$ Semiconductor Spintronics and Quantum Computation, edited by D. D. Awschalom, D. Loss, and N. Samarth (Springer-Verlag, Berlin, 2002).

${ }^{8}$ Y. Kato, R. C. Myers, A. C. Gossard, and D. D. Awschalom, Nature (London) 427, 50 (2004).

${ }^{9}$ J. M. Kikkawa and D. D. Awschalom, Nature (London) 397, 139 (1999).

${ }^{10}$ G. Salis, Y. Kato, K. Ensslin, D. C. Driscoll, A. C. Gossard, and D. D. Awschalom, Nature (London) 414, 619 (2001).

${ }^{11}$ I. E. Dzyaloshinkii, Sov. Phys. JETP 10, 628 (1959).

${ }^{12}$ L. S. Levitov, Y. V. Nazarov, and G. M. Éliasberg, Sov. Phys. JETP 61, 133 (1985).

${ }^{13}$ V. M. Edelstein, Phys. Rev. Lett. 75, 2004 (1995).

${ }^{14}$ A. G. Aronov and Y. B. Lyanda-Geller, JETP Lett. 50, 431 (1989)

${ }^{15}$ V. M. Edelstein, Solid State Commun. 73, 233 (1990).

${ }^{16}$ L. I. Magarill and M. V. Entin, JETP Lett. 72, 195 (2000).
${ }^{17}$ A. Voskoboynikov, S. S. Lin, C. P. Lee, and O. Tretyak, J. Appl. Phys. 87, 387 (2000).

${ }^{18}$ X. Cartoixà, D. Z.-Y. Ting, E. S. Daniel, and T. C. McGill, Superlattices Microstruct. 30, 309 (2001).

${ }^{19}$ Y. Kato, R. C. Myers, A. C. Gossard, and D. D. Awschalom, cond-mat/0403407 (unpublished).

${ }^{20}$ S. D. Ganichev, S. N. Danilov, P. Scheider, V. V. Bel'kov, L. E. Golub, W. Wegscheider, D. Weiss, and W. Prettl, cond-mat/ 0403641 (unpublished).

${ }^{21}$ C. M. Hurd, The Hall Effect in Metals and Alloys (Plenum, New York, 1973).

${ }^{22}$ The Hall effect and its Applications, edited by C. L. Chien and C. R. Westgate (Plenum, New York, 1980).

${ }^{23}$ M. I. Dyakonov and V. I. Perel, JETP Lett. 13, 467 (1971).

${ }^{24}$ J. E. Hirsch, Phys. Rev. Lett. 83, 1834 (1999).

${ }^{25}$ S. Murakami, N. Nagaosa, and S.-C. Zhang, Science 301, 1348 (2003).

${ }^{26}$ D. Culcer, J. Sinova, N. A. Sinitsyn, T. Jungwirth, A. H. MacDonald, and Q. Niu, cond-mat/0309475 (unpublished).

${ }^{27}$ B. A. Bernevig, J. Hu, E. Mukamel, and S.-C. Zhang, cond-mat/ 0311024 (unpublished).

${ }^{28}$ L. Hu, J. Gao, and S.-Q. Shen, cond-mat/0403231 (unpublished).

${ }^{29}$ J. Schliemann and D. Loss, cond-mat/0405436, Phys. Rev. B (to be published).

${ }^{30}$ J. Hu, B. A. Bernevig, and C. Wu, Int. J. Mod. Phys. B 17, 5991 (2003).

${ }^{31}$ S. Murakami, Phys. Rev. B 69, 241202(R) (2004). 
${ }^{32}$ J. Sinova, D. Culcer, Q. Niu, N. A. Sinitsyn, T. Jungwirth, and A. H. MacDonald, Phys. Rev. Lett. 92, 126603 (2004).

${ }^{33}$ J. Schliemann and D. Loss, Phys. Rev. B 69, 165315 (2004).

${ }^{34}$ N. A. Sinitsyn, E. M. Hankiewicz, W. Teizer, and J. Sinova, condmat/0310368 (unpublished).

${ }^{35}$ S.-Q. Shen, cond-mat/0310368 (unpublished).

${ }^{36}$ A. A. Burkov, A. S. Núñes, and A. H. MacDonald, cond-mat/ 0311328 (unpublished).

${ }^{37}$ E. I. Rashba, Phys. Rev. B 68, 241315(R) (2003).

${ }^{38}$ J. Inoue, G. E. W. Bauer, and L. W. Molenkamp, Phys. Rev. B 70, 041303(R) (2004).

${ }^{39}$ S.-Q. Shen, M. Ma, X. C. Xie, and F. C. Zhang, cond-mat/ 0403005 (unpublished).

${ }^{40}$ Y. Xiong and X. C. Xie, cond-mat/0403083 (unpublished).

${ }^{41}$ E. I. Rashba, Phys. Rev. B 70, 161201(R) (2004).

${ }^{42}$ O. Dimitrova, cond-mat/0405339 (unpublished).

${ }^{43}$ S. Murakami, cond-mat/0405003 (unpublished).

${ }^{44}$ L. W. Molenkamp, G. Schmidt, and G. E. W. Bauer, Phys. Rev. B 64, 121202(R) (2001).

${ }^{45}$ G. D. Mahan, Many-Particle Physics, 3rd ed. (Kluwer Academic, New York, 2000).

${ }^{46}$ J. B. Miller, D. M. Zumbühl, C. M. Marcus, Y. B. Lyanda-Geller, D. Goldhaber-Gordon, K. Campman, and A. C. Gossard, Phys. Rev. Lett. 90, 076807 (2003).

${ }^{47}$ R. Winkler, Phys. Rev. B 69, 045317 (2004).

${ }^{48}$ R. Winkler, cond-mat/0401106 (unpublished).

${ }^{49}$ D. Stein, K. von Klitzing, and G. Weinmann, Phys. Rev. Lett. 51, 130 (1983).

${ }^{50}$ M. Dobers, K. v. Klitzing, and G. Weimann, Phys. Rev. B 38, 5453 (1988).
${ }^{51}$ N. Nestle, G. Denninger, M. Vidal, C. Weinzierl, K. Brunner, K. Eberl, and K. von Klitzing, Phys. Rev. B 56, R4359 (1997).

${ }^{52}$ D. Grundler, Phys. Rev. Lett. 84, 6074 (2000).

${ }^{53}$ T. Koga, J. Nitta, T. Akazaki, and H. Takayanagi, Phys. Rev. Lett. 89, 046801 (2002).

${ }^{54}$ A. Brataas, Y. V. Nazarov, and G. E. W. Bauer, Eur. Phys. J. B 22, 99 (2001).

${ }^{55}$ Assuming that $v_{F} q \ll k_{B} T \ll \sqrt{\varepsilon_{\mathrm{SO}} \varepsilon_{F}}$, the dominant contribution comes from the region between the two Fermi contours (the spin-orbit contribution) if $\hbar \omega \gg \varepsilon_{F} q / k_{F}$.

${ }^{56}$ When Hamiltonians representing nonmagnetic impurites $H_{\mathrm{imp}}$ $=H_{\text {imp }}(\boldsymbol{r})$ are added to Eq. (1), the commutator in the Heisenberg equation of motion for an operator $O(\boldsymbol{r}, \boldsymbol{\sigma})$ becomes $\left[O(\boldsymbol{r}, \boldsymbol{\sigma}), H+H_{\mathrm{imp}}\right]=[O(\boldsymbol{r}, \boldsymbol{\sigma}), H]$, where $O$ is any operator that does not explicitly depend on $\boldsymbol{p}$. Both the particle and spin density are such operator and thus the equations of motion remain the same in the presence of impurities. The complications due to the impurities appear in the calculation of the average values of the operators.

${ }^{57}$ Homogeneous $(\boldsymbol{q}=0)$ spin systems driven by a constant field $E_{0}$ will exhibit damping of magnetization, i.e., $\partial_{t}\left\langle\rho_{\eta}(t \rightarrow \infty)\right\rangle=0$ for any finite amount of dissipation. Then, from Eq. (31) and the relation $\lim _{t \rightarrow \infty} \partial_{t}\left\langle\rho_{x}(t)\right\rangle=\lim _{s \rightarrow 0} s^{2}\left\langle\widetilde{\rho}_{x}(s)\right\rangle$ for the Laplace transform, we get $0=\lim _{s \rightarrow 0} s^{2} \widetilde{\rho}_{x}(s)=\left(2 m \beta E_{0} / \hbar^{2}\right) \widetilde{\sigma}_{x y}^{z}(s \rightarrow 0)$, where we used $\left\langle\widetilde{j}_{x}^{z}(s)\right\rangle=(\hbar / 2) \widetilde{\sigma}_{x y}^{z}(s) E_{0} s$ and $\alpha=0$. Thus, the dc spin conductivity vanishes for any dissipation mechanism. The same follows directly from the other magnetization components and in the case of $\alpha \neq 0$. 